\title{
Comprehensive Survey of PID Controller Design for the Inverted Pendulum
}

\author{
M. Kuczmann \\ Széchenyi István University, Department of Automation \\ Egyetem tér 1, H-9026, Gyốr, Hungary \\ E-mail: kuczmann@sze.hu
}

Abstract: The survey shows the detailed PID controller design how to stabilize the inclination angle as well as the horizontal movement of an inverted pendulum on a cart system step by step. Pendulum model is based on Euler-Lagrange modeling, and the nonlinear state space model is linearized in the unstable upward position. Controller design is performed by applying the transfer function description. The pendulum has been inserted into a virtual reality laboratory, which is suitable to use in model based control teaching.

Keywords: inverted pendulum, model based control, PID controller design

\section{Introduction}

The paper presents a comprehensive study of PID controller design for an inverted pendulum mounted on a cart.

The inverted pendulum is an unstable system that must be stabilized by the pushingpulling force $F=F(t)$ acting on the cart (Fig. 1), i.e. to reach $\varphi(t) \rightarrow 0$ in the stationary state, where $\varphi=\varphi(t)$ is the inclination angle. The pendulum simply falls over if the cart is not moved to balance it. The actuator is typically an electric motor. The cart can move only along the $x$-axis. 


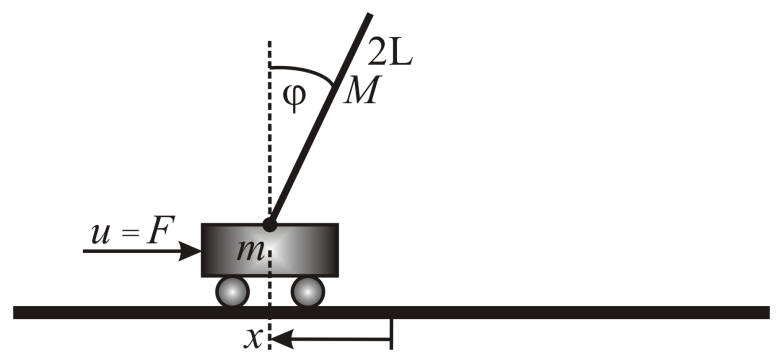

Figure 1. The inverted pendulum model.

The system input is the force, $u(t)=F(t)$. First, the output is only the inclination angle $\varphi$ resulting a single input single output system $(y(t)=\varphi(t))$. Second, the horizontal movement $x=x(t)$ is also stabilized, i.e. the plant is a single input and multiple output system $\left(y_{1}(t)=x(t), y_{2}(t)=\varphi(t)\right)$.

The studied plant is a popular example commonly found in control system textbooks and research literature [1-7]. The dynamics of the system are nonlinear as presented in the paper based on the above mentioned literature, but PID controller design is based on the linearized system. All the PID type controllers are presented in detail.

The aim of this survey paper is to show the Euler-Lagrange modeling of the inverted pendulum system, than the linear PID controller design step by step. The mentioned formulations are deeply presented in detail, that it why it can be used in teaching of model based control.

The really operating device has not built in this research, however the virtual reality based implementation has been performed which is applicable to understand the steps of control design.

A real-world example that relates directly to this inverted pendulum system is the attitude control of a booster rocket at takeoff or the well-known personal transporter Segway. 


\section{Dynamic model of the pendulum}

To set up the dynamic model of pendulum, the Euler-Lagrange equation is applied,

$$
\frac{\mathrm{d}}{\mathrm{d} t} \frac{\partial K}{\partial \dot{q}_{i}}-\frac{\partial K}{\partial q_{i}}+\frac{\partial P}{\partial \dot{q}_{i}}=\tau_{i},
$$

where $K$ is the kinetic energy, $P$ is the potential energy, $q_{i}$ and $\tau_{i}$ are the generalized coordinate and the generalized torque (force), respectively. In the case of pendulum $i=1,2$, i.e. $q_{1}=x$ and $q_{2}=\varphi$, moreover $\tau_{1}=F$ and $\tau_{2}=0$. Here $F$ is the force pulling or pushing the cart. This is an underactuated system because there are two output signals $(x$ and $\varphi$ ) and only one input signal $(F)$.

The kinetic energy of the system is as follows:

$$
K=\frac{1}{2} m \dot{x}^{2}+\frac{1}{2} M v_{M}^{2}+\frac{1}{2} \Theta \dot{\varphi}^{2},
$$

with the mass of cart, $m$, the mass of rod, $M$, and the inertial moment of the rod belonging to the center of mass, $\Theta$. The value of the last term is $\Theta=\frac{1}{3} M L^{2}$ (the length of the rod is $2 L$ ). The coordinates and the velocity of the center of mass of the rod are $x_{M}, y_{M}$ and $v_{M}$.

The potential energy of the system is

$$
P=M g L C_{\varphi}
$$

where $g$ is the gravitational acceleration. For simplicity, $S_{\varphi}=\sin \varphi$ and $C_{\varphi}=\cos \varphi$ notations are used in the paper.

The velocity of the rod center of mass can be obtained by the coordinates as follows:

$$
\begin{aligned}
v_{M}^{2}=\dot{x}_{M}^{2}+\dot{y}_{M}^{2} & =\left(\dot{x}+\left(L S_{\varphi}\right)^{\prime}\right)^{2}+\left(\left(L C_{\varphi}\right)^{\prime}\right)^{2} \\
& =\dot{x}^{2}+2 L C_{\varphi} \dot{x} \dot{\varphi}+L^{2} \dot{\varphi}^{2},
\end{aligned}
$$

since $x_{M}=x+L S_{\varphi}$ and $y_{M}=L C_{\varphi}$. 
The terms in (1) can be obtained easily:

$$
\begin{aligned}
& \frac{\partial K}{\partial \dot{x}}=m \dot{x}+M \dot{x}+M L C_{\varphi} \dot{\varphi}, \quad \frac{\partial K}{\partial x}=0, \quad \frac{\partial P}{\partial x}=0, \\
& \frac{\partial K}{\partial \dot{\varphi}}=M L C_{\varphi} \dot{x}+M L^{2} \dot{\varphi}+\Theta \dot{\varphi}, \quad \frac{\partial K}{\partial \varphi}=-M L S_{\varphi} \dot{x} \dot{\varphi}, \quad \frac{\partial P}{\partial \varphi}=-M g L S_{\varphi},
\end{aligned}
$$

and

$$
\begin{aligned}
\frac{\mathrm{d}}{\mathrm{d} t} \frac{\partial K}{\partial \dot{x}} & =m \ddot{x}+M \ddot{x}+M L C_{\varphi} \ddot{\varphi}-M L S_{\varphi} \dot{\varphi}^{2}, \\
\frac{\mathrm{d}}{\mathrm{d} t} \frac{\partial K}{\partial \dot{\varphi}} & =M L C_{\varphi} \ddot{x}-M L S_{\varphi} \dot{x} \dot{\varphi}+M L^{2} \ddot{\varphi}+\Theta \ddot{\varphi}
\end{aligned}
$$

Putting everything together gives the following differential equations:

$$
\begin{aligned}
& (m+M) \ddot{x}+M L C_{\varphi} \ddot{\varphi}-M L S_{\varphi} \dot{\varphi}^{2}=F, \\
& M L C_{\varphi} \ddot{x}+\left(M L^{2}+\Theta\right) \ddot{\varphi}-M g L S_{\varphi}=0 .
\end{aligned}
$$

From these equations, after some algebraic manipulations, the second order derivatives can be yielded as

$$
\ddot{\varphi}=\frac{M L S_{\varphi} C_{\varphi} \dot{\varphi}^{2}-(m+M) g S_{\varphi}+F C_{\varphi}}{M L C_{\varphi}^{2}-\frac{4}{3}(m+M) L},
$$

and

$$
\ddot{x}=\frac{\frac{4}{3} M L S_{\varphi} \dot{\varphi}^{2}-M g S_{\varphi} C_{\varphi}+\frac{4}{3} F}{\frac{4}{3}(m+M)-M C_{\varphi}^{2}} .
$$

The two second order differential equations can be rewritten as four first order differential equations by introducing state variables: $x_{1}=x, x_{2}=\dot{x}, x_{3}=\varphi$, $x_{4}=\dot{\varphi}$, i.e. $x_{2}=\dot{x}_{1}$ and $x_{4}=\dot{x}_{3}$. Finally, the state space representation of the 
dynamic modell is the following:

$$
\begin{aligned}
& \dot{x}_{1}=x_{2}, \\
& \dot{x}_{2}=\frac{\frac{4}{3} M L S_{x_{3}} \dot{x}_{4}^{2}-M g S_{x_{3}} C_{x_{3}}+\frac{4}{3} F}{\frac{4}{3}(m+M)-M C_{x_{3}}^{2}}, \\
& \dot{x}_{3}=x_{4}, \\
& \dot{x}_{4}=\frac{M L S_{x_{3}} C_{x_{3}} \dot{x}_{4}^{2}-(m+M) g S_{x_{3}}+F C_{x_{3}}}{M L C_{x_{3}}^{2}-\frac{4}{3}(m+M) L} .
\end{aligned}
$$

This nonlinear system can be linearized in the unstable upright position, when $\varphi=0$ and $\dot{\varphi}=0$, i.e. the approximations $S_{\varphi} \cong \varphi$ and $C_{\varphi} \cong 1$ can be applied.

At the end, the linearized system can be modelled by the following state space equations:

$$
\begin{aligned}
\dot{x}_{1} & =x_{2}, \\
\dot{x}_{2} & =\frac{-3 M g}{4 m+M} x_{3}+\frac{4}{4 m+M} F, \\
\dot{x}_{3} & =x_{4} \\
\dot{x}_{4} & =\frac{3(m+M) g}{4 m L+M L} x_{3}-\frac{3}{4 m L+M L} F .
\end{aligned}
$$

It can be written in the usual matrix form of SISO (single input single output) systems as

$$
\begin{aligned}
& \dot{\mathbf{x}}=\mathbf{A} \mathbf{x}+\mathbf{b} u, \\
& y=\mathbf{c}^{\mathrm{T}} \mathbf{x}+D u,
\end{aligned}
$$

where

$$
\mathbf{A}=\left[\begin{array}{cccc}
0 & 1 & 0 & 0 \\
0 & 0 & \frac{-3 M g}{4 m+M} & 0 \\
0 & 0 & 0 & 1 \\
0 & 0 & \frac{3(m+M) g}{4 m L+M L} & 0
\end{array}\right], \quad \mathbf{b}=\left[\begin{array}{c}
0 \\
\frac{4}{4 m+M} \\
0 \\
-\frac{3}{4 m L+M L}
\end{array}\right]
$$


Let the output $y$ of the system be the variable $\varphi$, in this case

$$
\mathbf{c}^{\mathrm{T}}=\left[\begin{array}{llll}
0 & 0 & 1 & 0
\end{array}\right], \quad D=0 .
$$

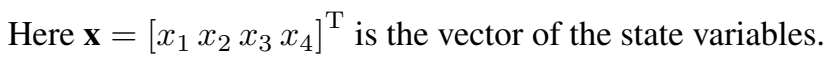

In the case of the two output system $\mathbf{y}=\mathbf{C x}+\mathbf{D} u$, where

$$
\mathbf{C}=\left[\begin{array}{llll}
1 & 0 & 0 & 0 \\
0 & 0 & 1 & 0
\end{array}\right], \quad \mathbf{D}=\left[\begin{array}{l}
0 \\
0
\end{array}\right],
$$

and $\mathbf{y}=[x \varphi]^{\mathrm{T}}$ is the output vector.

For simplicity, the following notations are used to obtain the transfer function of the model:

$$
\mathbf{A}=\left[\begin{array}{cccc}
0 & 1 & 0 & 0 \\
0 & 0 & q & 0 \\
0 & 0 & 0 & 1 \\
0 & 0 & p^{2} & 0
\end{array}\right], \quad \mathbf{b}=\left[\begin{array}{c}
0 \\
\beta \\
0 \\
\alpha
\end{array}\right]
$$

The transfer function of the system (13)-(14) with the notations defined by (16) is the following:

$$
W_{\mathrm{P}}(s)=\frac{\Phi(s)}{U(s)}=\frac{\mathcal{L}\{\varphi(t)\}}{\mathcal{L}\{u(t)\}}=\frac{\alpha}{s^{2}-p^{2}} .
$$

Here, the subscript $\mathrm{P}$ is for the plant, and the operator $\mathcal{L}\{\}$ represents the Laplace transform.

The transfer function

$$
W_{\mathrm{P}}(s)=\frac{-0.01}{s^{2}-1.47}=\frac{-0.01}{(s+1.21)(s-1.21)}
$$

is applied in the paper to represent the results ( $m=5, M=10,2 L=20$ in a coherent unit system, furthermore $\alpha=-0.01, p=1.21, \beta=0.13, q=-9.81$ ).

It is easy to see that the system is unstable, because one of the poles is surely positive: $p_{1,2}= \pm p$. It is noted that the four eigenvalues of the system matrix $\mathbf{A}$ are $\lambda_{1,2}=0, \lambda_{3,4}= \pm p$. 


\section{Stabilizing the SISO plant by PID controllers}

The typical closed loop block diagram of the controller system is shown in Fig. 2, where the Laplace transform of the signals are highlighted: the input and output of the plant are $U=U(s)$ and $\Phi=\Phi(s)$, the reference signal is zero, and $E=E(s)=$ $0-\Phi$ is the error.

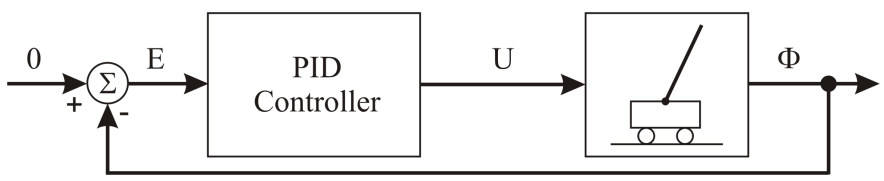

Figure 2. Block diagram of the controller system.

The transfer function of the open loop is $W_{\mathrm{O}}=W_{\mathrm{C}} W_{\mathrm{P}}$, where $W_{\mathrm{C}}$ and $W_{\mathrm{P}}$ are the transfer function of the controller and the plant, respectively. The notation $(s)$ will be cancelled in the following part of the paper.

In the followings, all the four controllers are studied.

\subsection{Applying $\mathbf{P}$ controller}

In the case of $\mathrm{P}$ controller, $W_{\mathrm{C}}$ is a constant, denoted by $K_{\mathrm{P}}\left(W_{\mathrm{C}}=K_{\mathrm{P}}\right)$, furthermore

$$
W_{\mathrm{O}}=K_{\mathrm{P}} \frac{\alpha}{s^{2}-p^{2}} \text {. }
$$

First, the Nyquist criterion and Nyquist plot are used to check whether the plant stabilization can be performed or not. It is well known that the Nyquist contour of this open loop system (with one unstable pole) should encircle counter clock-wise the point $-1+\mathrm{j} 0$ once. It can not be satisfied as it is demonstrated in Fig. 3 when the gain is negative (e.g. $K_{\mathrm{P}}=-200$ ). The Nyquist contour encircles the point $-1+\mathrm{j} 0$ once, but its direction is clock-wise. When $K_{\mathrm{P}}$ is positive, the Nyquist plot is on the right hand side plane resulting non stable system. 
The result can be verified by the root locus of the open loop transfer function (Fig. 3). One of the poles stands on the right hand side of the complex plane while $K_{\mathrm{P}} \geq 1$ is changing. It is not shown here, but the poles become unstable conjugate complex pairs when $K_{\mathrm{P}}$ is negative and large enough.
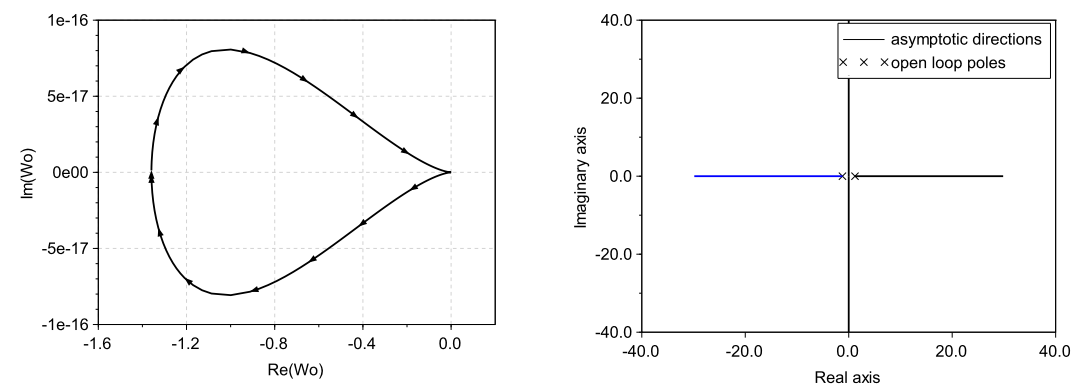

Figure 3. Nyquist plot and root locus of open loop system with P controller.

Now, let us prove this by analytically checking the poles of the closed loop system, too. The transfer function of the closed loop system is

$$
W_{\mathrm{CL}}=\frac{W_{\mathrm{O}}}{1+W_{\mathrm{O}}}=\frac{B}{A+B}
$$

if $W_{\mathrm{O}}=\frac{B}{A}$, moreover $B=B(s)$ and $A=A(s)$ are the numerator and the denominator of the open loop transfer function, respectively. The roots of the polynomial $A+B$ (the poles of $W_{\mathrm{CL}}$ ) are responsible for the stability of the closed loop system.

In the case of $\mathrm{P}$ controller $A+B=s^{2}-p^{2}+K_{\mathrm{P}} \alpha$, from which the poles are $p_{1,2}= \pm \sqrt{p^{2}-K_{\mathrm{P}} \alpha}$. If $K_{\mathrm{P}}$ is positive, one of the poles is usually unstable $(\alpha<0)$. If $K_{\mathrm{P}}$ is negative, the poles become complex numbers with zero real part.

At the end, it is concluded that stabilization can not be performed by simple $\mathrm{P}$ controller. 


\subsection{Applying PD controller}

In the case of PD controller,

$$
W_{\mathrm{C}}=K_{\mathrm{PD}} \frac{1+s T_{\mathrm{D}}}{1+s T_{\mathrm{D}}^{\prime}},
$$

where $T_{\mathrm{D}}$ and $T_{\mathrm{D}}^{\prime}$ are time constants, and $K_{\mathrm{PD}}$ is the gain of the controller.

First of all, $W_{\mathrm{P}}$ is rewritten in the form

$$
W_{\mathrm{P}}=\frac{\alpha}{s^{2}-p^{2}}=\frac{-\frac{\alpha}{p^{2}}}{\left(1+\frac{s}{p}\right)\left(1-\frac{s}{p}\right)} .
$$

Next, the stable pole of the plant is cancelled by $T_{\mathrm{D}}$, i.e. $T_{\mathrm{D}}=\frac{1}{p}$. The open loop transfer function becomes

$$
W_{\mathrm{O}}=-\frac{K_{\mathrm{PD}} \alpha}{p^{2}} \frac{1}{\left(1+s T_{\mathrm{D}}^{\prime}\right)\left(1-\frac{s}{p}\right)} .
$$

The following inequality must be satisfied when selecting the value of $T_{\mathrm{D}}^{\prime}$ :

$$
\frac{1}{T_{\mathrm{D}}^{\prime}}>\frac{1}{T_{\mathrm{D}}}
$$

The root locus of the open loop is shown first in Fig. 4 with positive and negative gain (here $T_{\mathrm{D}}^{\prime}=T_{\mathrm{D}} / 10$ is used). It is easy to see that the system can not be stabilized by any positive gain, but appropriate negative gain can stabilize the pendulum.

It can be checked by the Nyquist diagram of the open loop transfer function as well. See Fig. 5 for $K_{\mathrm{PD}}=-200$. The Nyquist contour encircles the point $-1+\mathrm{j} 0$ once counter clock-wise if the value of $K_{\mathrm{PD}}$ is large enough with negative sign.

Fig. 6 shows the impulse response of the closed loop system. The transient can oscillate when the gain is too high, anyway the stabilization time is shorter. 
M. Kuczmann - Acta Technica Jaurinensis, Vol.12, No.1, pp. 55-81, 2019
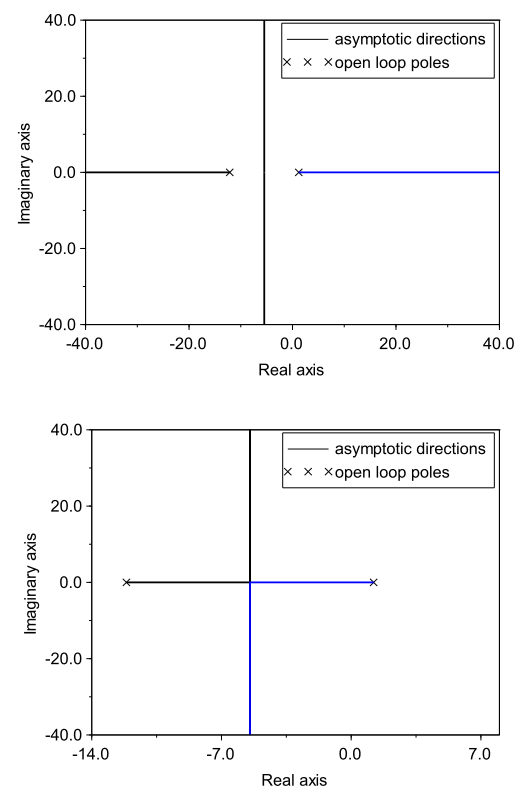

Figure 4. Open loop root locus of PD controller with positive and negative gain.

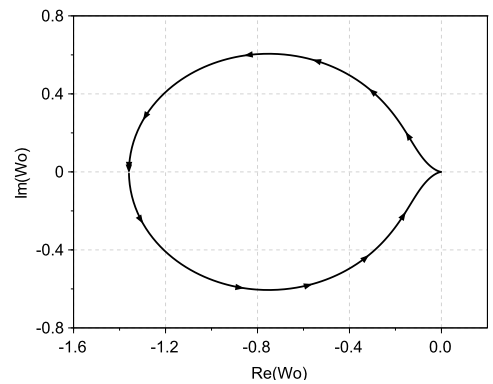

Figure 5. Nyquist plot of open loop system with PD controller. 
M. Kuczmann - Acta Technica Jaurinensis, Vol.12, No.1, pp. 55-81, 2019

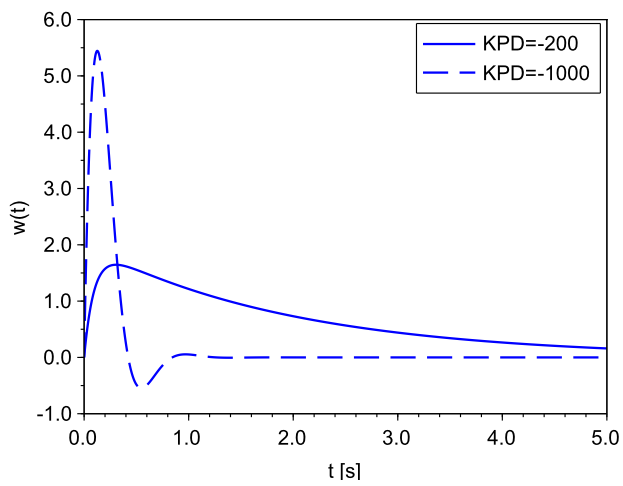

Figure 6. Impulse response of the closed loop system.
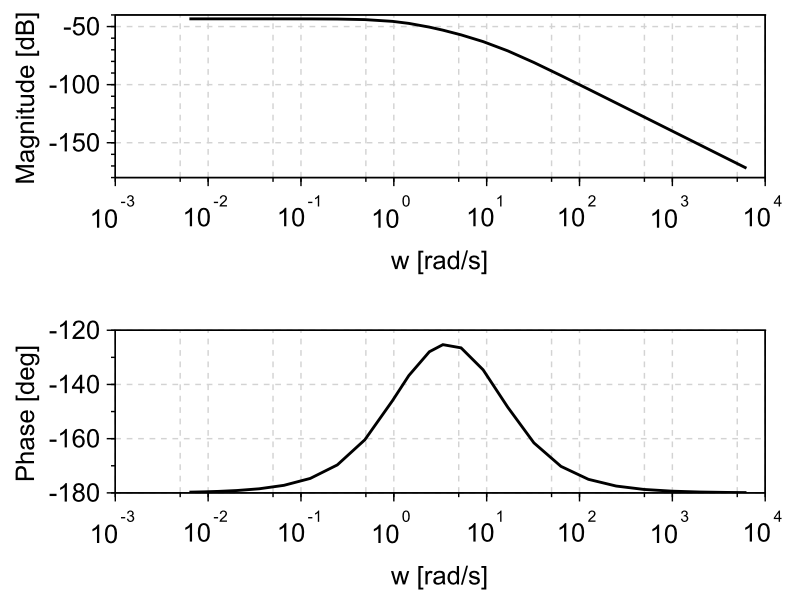

Figure 7. Bode plot of open loop system to determine gain. 
The open loop Bode plot is shown in Fig. 7, from which the maximum phase margin can be read easily, $P M_{\max } \cong 55^{\circ}$ at $\omega_{\mathrm{c}} \cong 3.8$, i.e. $K_{\mathrm{PD}}=-456$. In this case, there is no oscillation.

It can be computed analytically, too. The phase of the open loop system is the following:

$$
\Phi(\omega)=-180^{\circ}-\operatorname{atan} \omega T_{\mathrm{D}}^{\prime}+\operatorname{atan} \frac{\omega}{p}
$$

having the maximum value of phase margin at $\omega=\sqrt{\frac{p}{T_{\mathrm{D}}^{\prime}}}$, i.e. the maximum phase margin is

$$
P M_{\max }=-180^{\circ}-\operatorname{atan} \sqrt{p T_{\mathrm{D}}^{\prime}}+\operatorname{atan} \frac{1}{\sqrt{p T_{\mathrm{D}}^{\prime}}} .
$$

By these nonlinear expressions, the phase margin and the cut-off frequency, i.e. the transient behavior, can be designed semi-analytically.

For example, the PD controller

$$
W_{\mathrm{C}}=-456 \frac{1+s 0.82}{1+s 0.082}
$$

is a good choice to stabilize this system with maximum phase margin without oscillation.

Finally, the Routh-Hurwitz criterion is applied. In the case of PD controller $A+B=s^{2} T_{\mathrm{D}}^{\prime} p+s\left(p-p^{2} T_{\mathrm{D}}^{\prime}\right)+\left(K_{\mathrm{PD}} \alpha-p^{2}\right)$ which has the same roots as the monic polynomial $s^{2}+s \frac{\left(1-p T_{\mathrm{D}}^{\prime}\right)}{T_{\mathrm{D}}^{\prime}}+\frac{\left(K_{\mathrm{PD}} \alpha-p^{2}\right)}{T_{\mathrm{D}}^{\prime} p}$. According to the Routh-Hurwitz criterion, the coefficients of the above polynomials, after inserting the designed controller parameters must be positive.

PD controller can be designed by the Routh-Hurwitz criterion as well by prescribing the coefficients of the characteristic polynomial, i.e. by setting the value of $p_{1}$ and $p_{0}$,

$$
s^{2}+\underbrace{s T_{\mathrm{D}}^{\frac{\left(1-p T_{\mathrm{D}}^{\prime}\right)}{T^{\prime}}}}_{p_{1}}+\underbrace{\frac{\left(K_{\mathrm{PD}} \alpha-p^{2}\right)}{T_{\mathrm{D}}^{\prime} p}}_{p_{0}}=0,
$$


i.e.

$$
T_{\mathrm{D}}=\frac{1}{p}, \quad T_{\mathrm{D}}^{\prime}=\frac{1}{p+p_{1}}, \quad K_{\mathrm{PD}}=\frac{p^{2}+p_{0} p T_{\mathrm{D}}^{\prime}}{\alpha} .
$$

An example is presented here to show how to use these expressions. Let us, say, determine the overshoot of any transient to be less then $\Delta v_{\max }=5 \%$ and the settling time to be $T_{\mathrm{s}}=2$ with the tolerance fraction $\Delta=2 \%$. These criteria can be represented by supposing the dominant pole pair of the closed loop system, $p_{1,2}=-\xi \Omega \pm \mathrm{j} \Omega \sqrt{1-\xi^{2}}$, where $\xi$ and $\Omega$ are the damping ratio and the natural frequency of the complex pole pair. The damping ratio and the real part should be higher than $(\sigma=\xi \Omega)$

$$
\xi_{\min }=\frac{1}{\sqrt{1+\frac{\pi^{2}}{\ln ^{2} \Delta v_{\max }}}} \cong 0.7, \text { and } \quad \sigma_{\min }=-\frac{\ln \Delta}{T_{\mathrm{s}}} \cong 2 .
$$

The following dominant pole pair, for example, can satisfy these criteria $(\xi=0.9$, $\sigma=\xi \Omega=5): p_{1,2}=-5 \pm \mathrm{j} 2.4$, i.e. $p_{1}=10$ and $p_{0}=30.8$, and

$$
W_{\mathrm{C}}=-480 \frac{1+s 0.82}{1+s 0.089} .
$$

It can be concluded that the pendulum can be stabilized by a PD controller.

\subsection{Applying PI controller}

The transfer function of the PI controller is the following:

$$
W_{\mathrm{C}}=K_{\mathrm{PI}} \frac{1+s T_{\mathrm{I}}}{s T_{\mathrm{I}}},
$$

where $T_{\mathrm{I}}$ and $K_{\mathrm{PI}}$ are the time constant and the gain of the controller, respectively.

The stable pole of the plant is cancelled by $T_{\mathrm{I}}$, i.e. $T_{\mathrm{I}}=\frac{1}{p}$. The open loop transfer function can be written as

$$
W_{\mathrm{O}}=-\frac{K_{\mathrm{PI}} \alpha}{p^{2}} \frac{1}{s T_{\mathrm{I}}\left(1-\frac{s}{p}\right)} .
$$


It is easy to check that $A+B=s^{2}-s p+K_{\mathrm{PI}} \alpha$. The sign of the second term $-s p$ is usually negative, because $p>0$. It means that the Routh-Hurwitz criterion can not be satisfied, and the plant can not be stabilized by PI controller.

\subsection{Applying PID controller}

The following transfer function of PID controller has been used in this paper:

$$
W_{\mathrm{C}}=K_{\mathrm{PID}} \frac{1+s T_{\mathrm{I}}}{s T_{\mathrm{I}}} \frac{1+s T_{\mathrm{D}}}{1+s T_{\mathrm{D}}^{\prime}} .
$$

The stable pole of the plant is cancelled by $T_{\mathrm{D}}$, i.e. $T_{\mathrm{D}}=\frac{1}{p}$. The time constant of the integrator can also be set to the value $T_{\mathrm{I}}=\frac{1}{p}$. The inequality (24) must be satisfied again when selecting the value of $T_{\mathrm{D}}^{\prime}$. Finally, the open loop transfer function can be written as

$$
W_{\mathrm{O}}=-\frac{K_{\mathrm{PID}} \alpha}{p^{2}} \frac{1+\frac{s}{p}}{\frac{s}{p}} \frac{1}{\left(1+s T_{\mathrm{D}}^{\prime}\right)\left(1-\frac{s}{p}\right)} .
$$

Fig. 8 shows the root locus of the open loop system with negative gain when

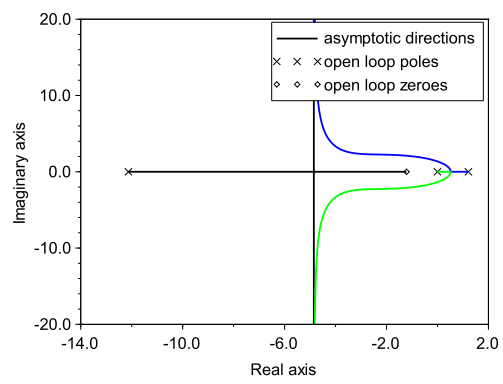

Figure 8. Root locus of open loop system with PID controller with negative gain. 
$T_{\mathrm{D}}^{\prime}=T_{\mathrm{D}} / 10$. It can be seen that the pendulum can be stabilized by a high enough negative gain. With positive gain it is not possible.

The maximum phase margin has been determined by the Bode plot resulting the gain $K_{\mathrm{PID}}=-871$ and the cut-off frequency $\omega_{\mathrm{c}} \cong 5.8$.

The open loop Nyquist diagram can be seen in Fig. 9. This is a magnified plot to check the counter clock-wise direction of the contour around the point $-1+\mathrm{j} 0$.

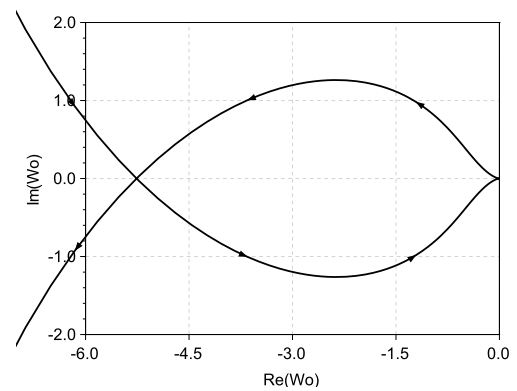

Figure 9. The open loop Nyquist diagram with PID controller, $K_{\mathrm{PID}}=-871$.

The PID controller

$$
W_{\mathrm{C}}=-871 \frac{1+s 0.82}{s 0.82} \frac{1+s 0.82}{1+s 0.082}
$$

is a good candidate to stabilize the system resulting the closed loop impulse response shown in Fig. 10.

Finally, the Routh-Hurwitz criterion is applied to check the stability. The following polynomial is obtained:

$$
A+B=s^{3} \underbrace{T_{\mathrm{D}}^{\prime} p}_{a_{3}}+s^{2} \underbrace{\left(p-p^{2} T_{\mathrm{D}}^{\prime}\right)}_{a_{2}}+s \underbrace{\left(K_{\mathrm{PID}} \alpha-p^{2}\right)}_{a_{1}}+\underbrace{K_{\mathrm{PID} \alpha p}}_{a_{0}}
$$

Not only the coefficients $a_{0}, a_{1}, a_{2}$ and $a_{3}$ must be positive but there is one more criterion according to the Routh-Hurwitz rule: $a_{1} a_{2}-a_{0} a_{3}$ must be positive. 


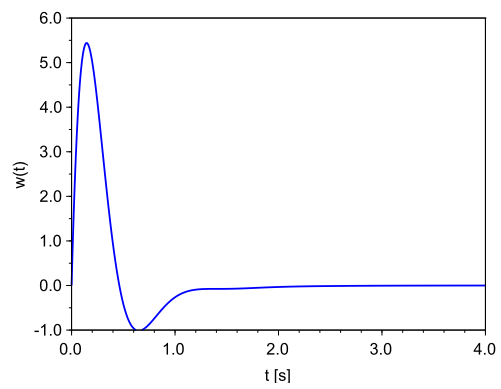

Figure 10. Impulse response of the closed loop system.

The controller can be designed by prescribing the coefficients of the characteristic polynomial, i.e. by setting the value of $p_{2}, p_{1}$ and $p_{0}$, moreover $T_{\mathrm{I}}$ is free,

$$
s^{3}+s^{2} \underbrace{\frac{1-p T_{\mathrm{D}}^{\prime}}{T_{\mathrm{D}}^{\prime}}}_{p_{2}}+s \underbrace{\frac{K_{\mathrm{PID}} \alpha-p^{2}}{p T_{\mathrm{D}}^{\prime}}}_{p_{1}}+\underbrace{\frac{K_{\mathrm{PID}} \alpha}{p T_{\mathrm{I}} T_{\mathrm{D}}^{\prime}}}_{p_{0}}=0,
$$

from which the controller parameters can be obtained as

$$
T_{\mathrm{D}}=\frac{1}{p}, \quad T_{\mathrm{D}}^{\prime}=\frac{1}{p+p_{2}}, \quad K_{\mathrm{PID}}=\frac{p^{2}+p_{1} T_{\mathrm{D}}^{\prime}}{\alpha}, \quad T_{\mathrm{I}}=\frac{\alpha K_{\mathrm{PID}}}{p p_{0} T_{\mathrm{D}}^{\prime}} .
$$

It can be concluded that PID controller is able to stabilize the pendulum.

\section{Stabilizing the horizontal movement}

Unfortunately, there is a problem with the above mentioned controllers: the cart moves along the $x$-axis, although the PD and the PID controllers are able to stabilize the angle of the pendulum. This design would not be feasible to implement on an actual physical system. In this Section not only the angle, but the horizontal movement is stabilized by PID controllers according to Fig. 11. 
The controll signal $u(t)$ is the sum of two controller signals [5], i.e.

$$
U=-W_{\mathrm{C}_{1}}\left(X_{\mathrm{r}}-X\right)+W_{\mathrm{C}_{2}}(0-\Phi) .
$$

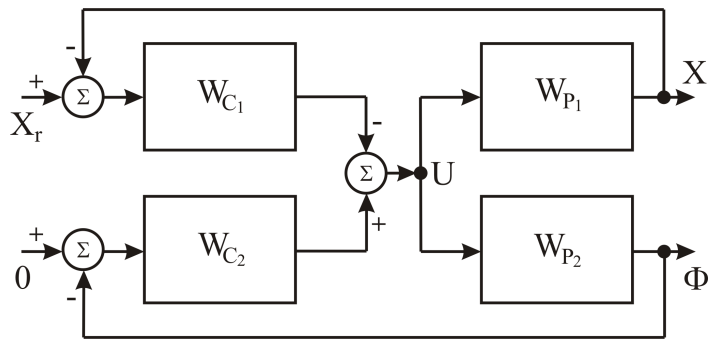

Figure 11. Block diagram of the two loop controller system.

The following transfer function with two integrators must be appended:

$$
W_{\mathrm{P}_{1}}=\frac{X}{U}=\frac{\beta s^{2}+\gamma}{s^{2}\left(s^{2}-p^{2}\right)},
$$

where $\gamma=\alpha q-\beta p^{2}$. The horizontal movement is according to the integrators in this transfer function.

In this section the following notation will be used for (17):

$$
W_{\mathrm{P}_{2}}(s)=\frac{\Phi}{U}=\frac{\alpha}{s^{2}-p^{2}} .
$$

The horizontal movement and the inclination angle can be expressed by the closed loop transfer functions,

$$
X=\frac{-W_{\mathrm{P}_{1}} W_{\mathrm{C}_{1}}}{1-W_{\mathrm{P}_{1}} W_{\mathrm{C}_{1}}+W_{\mathrm{P}_{2}} W_{\mathrm{C}_{2}}} X_{\mathrm{r}},
$$

and

$$
\Phi=\frac{-W_{\mathrm{P}_{2}} W_{\mathrm{C}_{1}}}{1-W_{\mathrm{P}_{1}} W_{\mathrm{C}_{1}}+W_{\mathrm{P}_{2}} W_{\mathrm{C}_{2}}} X_{\mathrm{r}}
$$


The closed loop system stability is depending on the denominator term 1 $W_{\mathrm{P}_{1}} W_{\mathrm{C}_{1}}+W_{\mathrm{P}_{2}} W_{\mathrm{C}_{2}}$. This means that the two controllers $W_{\mathrm{C}_{1}}$ and $W_{\mathrm{C}_{2}}$ can not be designed independently.

There are 16 possible combinations of the two controllers: P-P, P-PI, P-PD, P-PID, PI-P, PI-PI, PI-PD, PI-PID, PD-P, PD-PI, PD-PD, PD-PID, PID-P, PID-PI, PID-PD, PID-PID. Unfortunately, not all of these setups are feasible. Next, only the controller transfer functions mentioned in Section 3 are studied without analyzing the possible Diophantine equations [1,2] (this is planned in a separate paper). It is noted that the time constants have no got physical meaning, they are just parameters of the controller.

\subsection{P controller for horizontal movement}

In the case of P-P configuration $W_{\mathrm{C}_{1}}=K_{\mathrm{P}}^{1}, W_{\mathrm{C}_{2}}=K_{\mathrm{P}}^{2}$, i.e.

$$
1-W_{\mathrm{P}_{1}} W_{\mathrm{C}_{1}}+W_{\mathrm{P}_{2}} W_{\mathrm{C}_{2}}=1-\frac{\beta s^{2}+\gamma}{s^{2}\left(s^{2}-p^{2}\right)} K_{\mathrm{P}}^{1}+\frac{\alpha}{s^{2}-p^{2}} K_{\mathrm{P}}^{2}=0 .
$$

After a short algebra, the following polynomial can be obtained:

$$
s^{4}+0 s^{3}+\left(\alpha K_{\mathrm{P}}^{2}-\beta K_{\mathrm{P}}^{1}-p^{2}\right) s^{2}+0 s+\left(-K_{\mathrm{P}}^{1} \gamma\right),
$$

resulting a non stable closed loops system, because the coefficients of the terms $s^{3}$ and $s$ are zero.

It is easy to check the statement that, the P-PI system is similarly unstable. In this case $W_{\mathrm{C}_{1}}=K_{\mathrm{P}}^{1}, W_{\mathrm{C}_{2}}=K_{\mathrm{PI}}^{2} \frac{1+s T_{1}^{2}}{s T_{\mathrm{I}}^{2}}$, and the coefficient of the term $s^{3}$ is zero.

In the case of P-PD configuration $W_{\mathrm{C}_{1}}=K_{\mathrm{P}}^{1}, W_{\mathrm{C}_{2}}=K_{\mathrm{PD}}^{2} \frac{1+s T_{\mathrm{D}}^{2}}{1+s T_{\mathrm{D}}^{\prime 2}}$, i.e.

$$
1-W_{\mathrm{P}_{1}} W_{\mathrm{C}_{1}}+W_{\mathrm{P}_{2}} W_{\mathrm{C}_{2}}=1-\frac{\beta s^{2}+\gamma}{s^{2}\left(s^{2}-p^{2}\right)} K_{\mathrm{P}}^{1}+\frac{\alpha}{s^{2}-p^{2}} K_{\mathrm{PD}}^{2} \frac{1+s T_{\mathrm{D}}^{2}}{1+s T_{\mathrm{D}}^{\prime 2}}=0,
$$


from which, after a short algebra, the following polynomial can be given:

$$
\begin{aligned}
& s^{5}+\frac{1}{T_{\mathrm{D}}^{\prime 2}} s^{4}+\left(-p^{2}-\beta K_{\mathrm{P}}^{1}+\alpha K_{\mathrm{PD}}^{2} \frac{T_{\mathrm{D}}^{2}}{T_{\mathrm{D}}^{\prime 2}}\right) s^{3}+\frac{-p^{2}-\beta K_{\mathrm{P}}^{1}+\alpha K_{\mathrm{PD}}^{2}}{T_{\mathrm{D}}^{\prime 2}} s^{2} \\
& \quad+\left(-\gamma K_{\mathrm{P}}^{1}\right) s+\left(-\frac{\gamma K_{\mathrm{P}}^{1}}{T_{\mathrm{D}}^{\prime 2}}\right) .
\end{aligned}
$$

The P-PD configuration is redundant, because there are four parameters to be determined $\left(K_{\mathrm{P}}^{1}, K_{\mathrm{PD}}^{2}, T_{\mathrm{D}}^{2}, T_{\mathrm{D}}^{\prime 2}\right)$, but there are five equations according to the fifth order polynomial. In the followings, redundant systems will be skipped.

The P-PID system is finally feasible. From the characteristic equation

$$
1-\frac{\beta s^{2}+\gamma}{s^{2}\left(s^{2}-p^{2}\right)} K_{\mathrm{P}}^{1}+\frac{\alpha}{s^{2}-p^{2}} K_{\mathrm{PID}}^{2} \frac{1+s T_{\mathrm{I}}^{2}}{s T_{\mathrm{I}}^{2}} \frac{1+s T_{\mathrm{D}}^{2}}{1+s T_{\mathrm{D}}^{\prime 2}}=0
$$

the following polynomial can be got:

$$
\begin{aligned}
s^{5} & +\underbrace{\frac{1}{T_{\mathrm{D}}^{\prime 2}}}_{p_{4}} s^{4}+\underbrace{\left(-p^{2}-\beta K_{\mathrm{P}}^{1}\right)}_{p_{3}} s^{3}+\underbrace{\frac{-p^{2}-\beta K_{\mathrm{P}}^{1}+\alpha K_{\mathrm{PID}}^{2} T_{\mathrm{D}}^{2}}{T_{\mathrm{D}}^{\prime 2}}}_{p_{2}} s^{2} \\
+ & \underbrace{\left(-\gamma K_{\mathrm{P}}^{1}+\alpha K_{\mathrm{PID}}^{2} \frac{T_{\mathrm{D}}^{2}}{T_{\mathrm{I}}^{2} T_{\mathrm{D}}^{\prime 2}}+\alpha K_{\mathrm{PID}}^{2} \frac{1}{T_{\mathrm{D}}^{\prime 2}}\right)}_{p_{1}} s+\underbrace{\frac{\alpha K_{\mathrm{PID}}^{2}-\gamma K_{\mathrm{P}}^{1} T_{\mathrm{I}}^{2}}{T_{\mathrm{I}}^{2} T_{\mathrm{D}}^{\prime 2}}}_{p_{0}} .
\end{aligned}
$$

From the resulting five equations the following controller parameters can be obtained analytically:

$$
T_{\mathrm{D}}^{\prime 2}=\frac{1}{p_{4}}, \quad K_{\mathrm{P}}^{1}=-\frac{p_{0} T_{\mathrm{D}}^{\prime 2}}{\gamma},
$$

and $T_{\mathrm{D}}^{2}$ is the solution of the second order equation

$$
\left(p_{1}+\gamma K_{\mathrm{P}}^{1}\right) T_{\mathrm{D}}^{\prime 2}\left(T_{\mathrm{D}}^{2}\right)^{2}+\left(-p^{2}-\beta K_{\mathrm{P}}^{1}-p_{2} T_{\mathrm{D}}^{\prime 2}\right) T_{\mathrm{D}}^{2}+\left(p_{3}+p^{2}+\beta K_{\mathrm{P}}^{1}\right) T_{\mathrm{D}}^{\prime 2}=0,
$$

finally

$$
T_{\mathrm{I}}^{2}=\frac{p_{3}+p^{2}+\beta K_{\mathrm{P}}^{1}}{\left(p_{1}+\gamma K_{\mathrm{P}}^{1}\right) T_{\mathrm{D}}^{2}}, \quad K_{\mathrm{PID}}^{2}=\frac{p_{1}+\gamma K_{\mathrm{P}}^{1}}{\alpha} T_{\mathrm{I}}^{2} T_{\mathrm{D}}^{\prime 2} .
$$


The impulse response of this system can be seen in Fig. 12 when all the desired poles of the closed loop system are equal to -1 , i.e. the characteristic polynomial is prescribed by $s^{5}+5 s^{4}+10 s^{3}+10 s^{2}+5 s+1$. It can be seen that all the system outputs have been stabilized. The behavior of the closed loop system can be set by the desired poles.
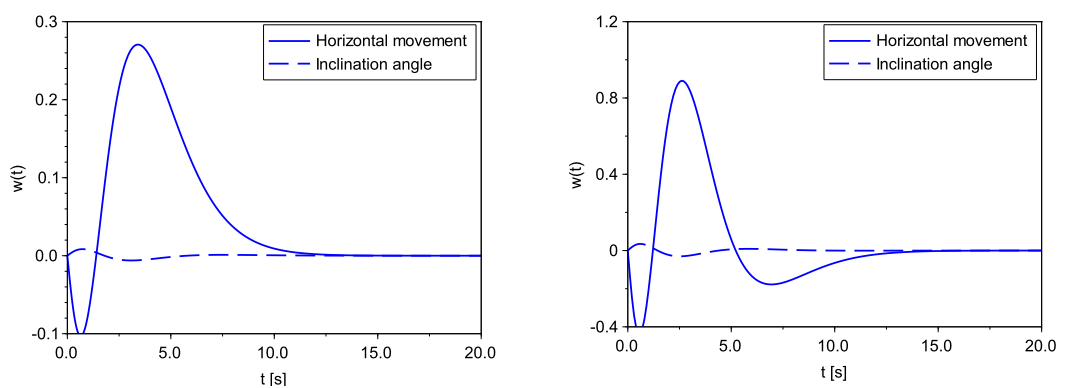

Figure 12. Impulse response of the closed loop P-PID and PI-PID systems.

\subsection{PI controller for horizontal movement}

Only PI-PID can be used as stabilizing controller from the second group. It is easy to check that, configurations PI-P, PI-PI and PI-PD result in redundant systems, moreover some of the polynomial coefficients are equal to zero.

The design of a feasible PI-PID system can be performed by the characteristic equation

$$
1-\frac{\beta s^{2}+\gamma}{s^{2}\left(s^{2}-p^{2}\right)} K_{\mathrm{PI}}^{1} \frac{1+s T_{\mathrm{I}}^{1}}{s T_{\mathrm{I}}^{1}}+\frac{\alpha}{s^{2}-p^{2}} K_{\mathrm{PID}}^{2} \frac{1+s T_{\mathrm{I}}^{2}}{s T_{\mathrm{I}}^{2}} \frac{1+s T_{\mathrm{D}}^{2}}{1+s T_{\mathrm{D}}^{\prime 2}}=0 .
$$


The following polynomial can be obtained after some manipulations:

$$
\begin{aligned}
& s^{6}+\underbrace{\frac{1}{T_{\mathrm{D}}^{\prime 2}}}_{p_{5}} s^{5}+\underbrace{\left(-p^{2}-\beta K_{\mathrm{PI}}^{1}+\alpha K_{\mathrm{PID}}^{2} \frac{T_{\mathrm{D}}^{2}}{T_{\mathrm{D}}^{\prime 2}}\right)}_{p_{4}} s^{4} \\
& +\underbrace{\left(-\frac{p^{2}}{T_{\mathrm{D}}^{\prime 2}}-\beta K_{\mathrm{PI}}^{1} \frac{T_{\mathrm{I}}^{1}+T_{\mathrm{D}}^{\prime 2}}{T_{\mathrm{I}}^{1} T_{\mathrm{D}}^{\prime 2}}+\alpha K_{\mathrm{PID}}^{2} \frac{T_{\mathrm{I}}^{2}+T_{\mathrm{D}}^{2}}{T_{\mathrm{I}}^{2} T_{\mathrm{D}}^{\prime 2}}\right)}_{p_{3}} s^{3} \\
& +\underbrace{\left(-\gamma K_{\mathrm{PI}}^{1}-\beta K_{\mathrm{PI}}^{1} \frac{1}{T_{\mathrm{I}}^{1} T_{\mathrm{D}}^{\prime 2}}+\alpha K_{\mathrm{PID}}^{2} \frac{1}{T_{\mathrm{I}}^{2} T_{\mathrm{D}}^{\prime 2}}\right)}_{p_{2}} s^{2} \\
& +\underbrace{\left(-\gamma K_{\mathrm{PI}}^{1} \frac{T_{\mathrm{I}}^{1}+T_{\mathrm{D}}^{\prime 2}}{T_{\mathrm{I}}^{1} T_{\mathrm{D}}^{\prime 2}}\right)}_{p_{1}} s+\underbrace{\frac{-\gamma K_{\mathrm{PI}}^{1}}{T_{\mathrm{I}}^{1} T_{\mathrm{D}}^{\prime 2}}}_{p_{0}} .
\end{aligned}
$$

From the resulting six equations the following controller parameters can be obtained analytically:

$$
T_{\mathrm{D}}^{\prime 2}=\frac{1}{p_{5}}, \quad T_{\mathrm{I}}^{1}=\frac{p_{1}}{p_{0}}-T_{\mathrm{D}}^{\prime 2}, \quad K_{\mathrm{PI}}^{1}=-\frac{p_{0} T_{\mathrm{I}}^{1} T_{\mathrm{D}}^{\prime 2}}{\gamma},
$$

the parameter $T_{\mathrm{D}}^{2}$ is the solution of the second order equation

$$
\begin{aligned}
\left(p_{2}+\gamma K_{\mathrm{PI}}^{1}+\frac{\beta K_{\mathrm{PI}}^{1}}{T_{\mathrm{I}}^{1} T_{\mathrm{D}}^{\prime 2}}\right)\left(T_{\mathrm{D}}^{2}\right)^{2} & +\left(-p_{3}-\frac{p^{2}}{T_{\mathrm{D}}^{\prime 2}}-\beta K_{\mathrm{PI}}^{1} \frac{T_{\mathrm{I}}^{1}+T_{\mathrm{D}}^{\prime 2}}{T_{\mathrm{I}}^{1} T_{\mathrm{D}}^{\prime 2}}\right) T_{\mathrm{D}}^{2} \\
& +\left(p_{4}+p^{2}+\beta K_{\mathrm{PI}}^{1}\right)=0,
\end{aligned}
$$

and finally

$$
T_{\mathrm{I}}^{2}=\frac{p_{4}+p^{2}+\beta K_{\mathrm{PI}}^{1}}{T_{\mathrm{D}}^{2}\left(p_{2}+\gamma K_{\mathrm{PI}}^{1}+\frac{\beta K_{\mathrm{PI}}^{1}}{T_{\mathrm{I}}^{1} T_{\mathrm{D}}^{\prime 2}}\right)}, \quad K_{\mathrm{PID}}^{2}=\frac{p_{4}+p^{2}+\beta K_{\mathrm{PI}}^{1}}{\alpha T_{\mathrm{D}}^{2}} T_{\mathrm{D}}^{\prime 2} .
$$

The impulse response of the stabilized system can also be seen in Fig. 12. All the closed loop system poles have been set to -1 , i.e. the characteristic polynomial is prescribed by $s^{6}+6 s^{5}+15 s^{4}+20 s^{3}+15 s^{2}+6 s+1$. 


\subsection{PD controller for horizontal movement}

When $W_{\mathrm{C}_{1}}=K_{\mathrm{PD}}^{1} \frac{1+s T_{\mathrm{D}}^{1}}{1+s T_{\mathrm{D}}^{\prime 1}}$, PD-PI and PD-PD controllers result in feasible solution. PD-P controller is redundant and, at the same time, PD-PID is under determined, because there are seven parameters to be determined, but the characteristic polynomial degree is only six.

The PD-PI controller is based on the equation:

$$
1-\frac{\beta s^{2}+\gamma}{s^{2}\left(s^{2}-p^{2}\right)} K_{\mathrm{PD}}^{1} \frac{1+s T_{\mathrm{D}}^{1}}{1+s T_{\mathrm{D}}^{\prime 1}}+\frac{\alpha}{s^{2}-p^{2}} K_{\mathrm{PI}}^{2} \frac{1+s T_{\mathrm{I}}^{2}}{s T_{\mathrm{I}}^{2}}=0,
$$

from which the following polynomial can be written:

$$
\begin{gathered}
s^{5}+\underbrace{\frac{1}{T_{\mathrm{D}}^{\prime 1}}}_{p_{4}} s^{4}+\underbrace{\left(-p^{2}-\beta K_{\mathrm{PD}}^{1} \frac{T_{\mathrm{D}}^{1}}{T_{\mathrm{D}}^{\prime 1}}+\alpha K_{\mathrm{PI}}^{2}\right)}_{p_{3}} s^{3} \\
+\underbrace{\left(-\frac{p^{2}}{T_{\mathrm{D}}^{\prime 1}}-\frac{\beta K_{\mathrm{PD}}^{1}}{T_{\mathrm{D}}^{\prime 1}}+\alpha K_{\mathrm{PI}}^{2} \frac{T_{\mathrm{D}}^{\prime 1}+T_{\mathrm{I}}^{2}}{T_{\mathrm{D}}^{\prime 1} T_{\mathrm{I}}^{2}}\right)}_{p_{2}} s^{2} \\
+\underbrace{\left(\frac{\alpha K_{\mathrm{PI}}^{2}}{T_{\mathrm{D}}^{\prime 1} T_{\mathrm{I}}^{2}}-\frac{\gamma K_{\mathrm{PD}}^{1} T_{\mathrm{D}}^{1}}{T_{\mathrm{D}}^{\prime 1}}\right)}_{p_{1}} s+\underbrace{\left(-\frac{\gamma K_{\mathrm{PD}}^{1}}{T_{\mathrm{D}}^{\prime 1}}\right)}_{p_{0}} .
\end{gathered}
$$

The analytical solution of the five equations is quite simple:

$$
\begin{aligned}
T_{\mathrm{D}}^{\prime 1} & =\frac{1}{p_{4}}, \quad K_{\mathrm{PD}}^{1}=-\frac{p_{0} T_{\mathrm{D}}^{\prime 1}}{\gamma}, \quad T_{\mathrm{D}}^{1}=\frac{p_{2} T_{\mathrm{D}}^{\prime 1}-p_{1}\left(T_{\mathrm{D}}^{\prime 1}\right)^{2}-p_{3}+\beta K_{\mathrm{PD}}^{1}}{\frac{\beta K_{\mathrm{PD}}^{1}}{T_{\mathrm{D}}^{\prime 1}}+\gamma K_{\mathrm{PD}}^{1} T_{\mathrm{D}}^{\prime 1}}, \\
T_{\mathrm{I}}^{2} & =\frac{p_{2} T_{\mathrm{D}}^{\prime 1}-p_{1}\left(T_{\mathrm{D}}^{\prime 1}\right)^{2}-\gamma K_{\mathrm{PD}}^{1} T_{\mathrm{D}}^{1} T_{\mathrm{D}}^{\prime 1}+p^{2}+\beta K_{\mathrm{PD}}^{1}}{\gamma K_{\mathrm{PD}}^{1} T_{\mathrm{D}}^{1}+p_{1} T_{\mathrm{D}}^{\prime 1}}, \\
K_{\mathrm{PI}}^{2} & =\frac{p_{1} T_{\mathrm{D}}^{\prime 1}+\gamma K_{\mathrm{PD}}^{1} T_{\mathrm{D}}^{1}}{\alpha} T_{\mathrm{I}}^{2} .
\end{aligned}
$$


The PD-PD controller is based on the equation:

$$
1-\frac{\beta s^{2}+\gamma}{s^{2}\left(s^{2}-p^{2}\right)} K_{\mathrm{PD}}^{1} \frac{1+s T_{\mathrm{D}}^{1}}{1+s T_{\mathrm{D}}^{\prime 1}}+\frac{\alpha}{s^{2}-p^{2}} K_{\mathrm{PD}}^{2} \frac{1+s T_{\mathrm{D}}^{2}}{1+s T_{\mathrm{D}}^{\prime 2}}=0 .
$$

The following characteristic polynomial is coming out after some mathematical manipulations:

$$
\begin{aligned}
& s^{6}+s^{5} \underbrace{\frac{T_{\mathrm{D}}^{\prime 1}+T_{\mathrm{D}}^{\prime 2}}{T_{\mathrm{D}}^{\prime 1} T_{\mathrm{D}}^{\prime 2}}}_{p_{5}}+s^{4} \underbrace{\left(\frac{1}{T_{\mathrm{D}}^{\prime 1} T_{\mathrm{D}}^{\prime 2}}-\beta K_{\mathrm{PD}}^{1} \frac{T_{\mathrm{D}}^{1}}{T_{\mathrm{D}}^{\prime 1}}+\alpha K_{\mathrm{PD}}^{2} \frac{T_{\mathrm{D}}^{2}}{T_{\mathrm{D}}^{\prime 2}}-p^{2}\right)}_{p_{4}} \\
& +s^{3} \underbrace{\left(-p^{2} \frac{T_{\mathrm{D}}^{\prime 1}+T_{\mathrm{D}}^{\prime 2}}{T_{\mathrm{D}}^{\prime 2} T_{\mathrm{D}}^{\prime 2}}-\beta K_{\mathrm{PD}}^{1} \frac{T_{\mathrm{D}}^{1}+T_{\mathrm{D}}^{\prime 2}}{T_{\mathrm{D}}^{\prime 1} T_{\mathrm{D}}^{\prime 2}}+\alpha K_{\mathrm{PD}}^{2} \frac{T_{\mathrm{D}}^{\prime 1}+T_{\mathrm{D}}^{2}}{T_{\mathrm{D}}^{\prime 1} T_{\mathrm{D}}^{\prime 2}}\right)}_{p_{3}} \\
& +s^{2} \underbrace{\left(-\frac{p^{2}}{T_{\mathrm{D}}^{\prime 1} T_{\mathrm{D}}^{\prime 2}}-\beta K_{\mathrm{PD}}^{1} \frac{1}{T_{\mathrm{D}}^{\prime 1} T_{\mathrm{D}}^{\prime 2}}-\gamma K_{\mathrm{PD}}^{1} \frac{T_{\mathrm{D}}^{1}}{T_{\mathrm{D}}^{\prime 1}}+\alpha K_{\mathrm{PD}}^{2} \frac{1}{T_{\mathrm{D}}^{\prime 1} T_{\mathrm{D}}^{\prime 2}}\right)}_{p_{1}} \\
& +s \underbrace{\left(-\gamma K_{\mathrm{PD}}^{1} \frac{T_{\mathrm{D}}^{1}+T_{\mathrm{D}}^{\prime 2}}{T_{\mathrm{D}}^{\prime 1} T_{\mathrm{D}}^{\prime 2}}\right)}_{p_{2}}+\underbrace{\left(-\frac{\gamma K_{\mathrm{PD}}^{1}}{T_{\mathrm{D}}^{\prime 1} T_{\mathrm{D}}^{\prime 2}}\right)}_{p_{0}} .
\end{aligned}
$$

The analytical solution of the equations according to this polynomial is tedious. Now, the system of six nonlinear equations has been solved numerically. In this case it is very difficult to find an initial set of the unknown parameters. The experience of this study is that, it is much convenient to use analytically solvable controllers.

\subsection{PID controller for horizontal movement}

The PID-P controller is redundant, but the other three versions can stabilize the pendulum. The PID-PI controller setup easily can be obtained analytically. The design of a feasible system is based on the characteristic equation

$$
1-\frac{\beta s^{2}+\gamma}{s^{2}\left(s^{2}-p^{2}\right)} K_{\mathrm{PID}}^{1} \frac{1+s T_{\mathrm{I}}^{1}}{s T_{\mathrm{I}}^{1}} \frac{1+s T_{\mathrm{D}}^{1}}{1+s T_{\mathrm{D}}^{\prime 1}}+\frac{\alpha}{s^{2}-p^{2}} K_{\mathrm{PI}}^{2} \frac{1+s T_{\mathrm{I}}^{2}}{s T_{\mathrm{I}}^{2}}=0,
$$


from which

$$
\begin{aligned}
& s^{6}+\underbrace{\frac{1}{T_{\mathrm{D}}^{\prime 1}}}_{p_{5}} s^{5}+\underbrace{\left(-p^{2}-\beta K_{\mathrm{PID}}^{1} \frac{T_{\mathrm{D}}^{1}}{T_{\mathrm{D}}^{\prime 1}}+\alpha K_{\mathrm{PI}}^{2}\right)}_{p_{4}} s^{4} \\
& +\underbrace{\left(-\frac{p^{2}}{T_{\mathrm{D}}^{\prime 1}}-\beta K_{\mathrm{PID}}^{1} \frac{T_{\mathrm{I}}^{1}+T_{\mathrm{D}}^{1}}{T_{\mathrm{I}}^{1} T_{\mathrm{D}}^{\prime 1}}+\alpha K_{\mathrm{PI}}^{2} \frac{T_{\mathrm{I}}^{2}+T_{\mathrm{D}}^{\prime 1}}{T_{\mathrm{I}}^{2} T_{\mathrm{D}}^{\prime 1}}\right)}_{p_{3}} s^{3} \\
& +\underbrace{\left(-\gamma K_{\mathrm{PID}}^{1} \frac{T_{\mathrm{D}}^{1}}{T_{\mathrm{D}}^{\prime 1}}-\beta K_{\mathrm{PID}}^{1} \frac{1}{T_{\mathrm{I}}^{1} T_{\mathrm{D}}^{\prime 1}}+\alpha K_{\mathrm{PI}}^{2} \frac{1}{T_{\mathrm{I}}^{2} T_{\mathrm{D}}^{\prime 1}}\right)}_{p_{2}} s^{2} \\
& +\underbrace{\left(-\gamma K_{\mathrm{PID}}^{1} \frac{T_{\mathrm{I}}^{1}+T_{\mathrm{D}}^{1}}{T_{\mathrm{I}}^{1} T_{\mathrm{D}}^{\prime 1}}\right)}_{p_{1}} s+\underbrace{\frac{-\gamma K_{\mathrm{PID}}^{1}}{T_{\mathrm{I}}^{1} T_{\mathrm{D}}^{\prime 1}}}_{p_{0}}
\end{aligned}
$$

is the characteristic polynomial, where from the controller parameters can be obtained analytically, $T_{\mathrm{D}}^{\prime 1}=\frac{1}{p_{5}}$, furthermore the parameter $T_{\mathrm{D}}^{1}$ is the solution of the second order equation

$$
\begin{aligned}
\left(\frac{\beta p_{0}}{\gamma T_{\mathrm{D}}^{\prime 1}}+p_{0} T_{\mathrm{D}}^{\prime 1}\right)\left(T_{\mathrm{D}}^{1}\right)^{2} & +\left(-\frac{\beta p_{1}}{\gamma T_{\mathrm{D}}^{\prime 1}}-p_{1} T_{\mathrm{D}}^{\prime 1}\right) T_{\mathrm{D}}^{1} \\
& +\left(\frac{\beta p_{1}}{\gamma}-\frac{\beta p_{0}}{\gamma} T_{\mathrm{D}}^{\prime 1}+\frac{p_{4}}{T_{\mathrm{D}}^{\prime 1}}+p_{2} T_{\mathrm{D}}^{\prime 1}-p_{3}\right)=0,
\end{aligned}
$$

and finally

$$
\begin{aligned}
& T_{\mathrm{I}}^{1}=\frac{p_{1}}{p_{0}}-T_{\mathrm{D}}^{1}, \quad K_{\mathrm{PID}}^{1}=-\frac{p_{0} T_{\mathrm{I}}^{1} T_{\mathrm{D}}^{\prime 1}}{\gamma}, \quad K_{\mathrm{PI}}^{2}=\frac{p_{4}+p^{2}+\beta K_{\mathrm{PID}}^{1} \frac{T_{\mathrm{D}}^{1}}{T_{\mathrm{D}}^{\prime 1}}}{\alpha}, \\
& T_{\mathrm{I}}^{2}=\frac{p_{4}+p^{2}+\frac{\beta p_{0}}{\gamma}\left(T_{\mathrm{D}}^{1}\right)^{2}-\frac{\beta p_{1}}{\gamma} T_{\mathrm{D}}^{1}}{p_{2} T_{\mathrm{D}}^{\prime 1}-p_{1} T_{\mathrm{D}}^{1} T_{\mathrm{D}}^{\prime 1}+p_{0} T_{\mathrm{D}}^{\prime 1}\left(T_{\mathrm{D}}^{1}\right)^{2}-\frac{\beta p_{0}}{\gamma} T_{\mathrm{D}}^{\prime 1}} .
\end{aligned}
$$

The other two controllers, PID-PD and PID-PID, contain seven and eight parameters, respectively, and the characteristic polynomial order is seven. The detailed 
presentation of these equations are not shown here. The analytical solution of the equations according to these controllers is lengthy and tedious, the system of nonlinear equations can be solved numerically in both cases. It must be highlighted again that, it is very difficult to find a good initial set of the unknown parameters. Anyway it is not a drawback, because there are many other possibilities that can stabilize the pendulum as it is previously shown.

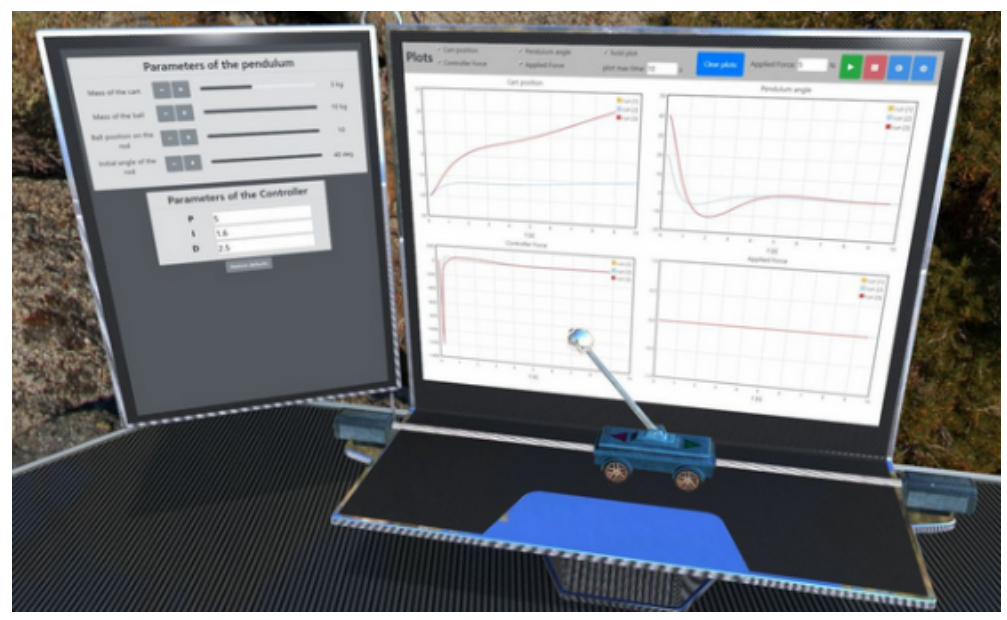

Figure 13. The inverted pendulum model in the virtual laboratory of MaxWhere.

\section{Implementation}

Controller design and analysis have been realized firstly in Scilab [8]. Then the virtual reality based implementation has been performed in the frame of MaxWhere as a freely available virtual laboratory $[9,10]$. A separate future paper is planned to show the virtual lab. A snapshot about the lab can be seen in Fig. 13, where the controller settings and oscilloscopes showing some signals (e.g. the angle of pendulum, the horizontal position, the acting force versus the time) can be seen among other information like the theoretical background. 


\section{Conclusion and future work}

Classical PID controller design for the problem of inverted pendulum has been shown in detail in this paper. Two controllers are necessary to design for stabilizing the system. It is clear that, the design of two dependent controllers is tedious in some cases, however the state feedback controllers can solve this problem in an easy way. Next, the state space representation based controllers will be presented [1-4,6,7], like the Ackermann formula and the Bass-Gura pole placement techniques, furthermore the linear quadratic optimal controller design. The Diophantine equation based controller design, the Youla-parametrization [1] are also planned to realize as well as nonlinear techniques $[11,12]$ and model predictive control [13].

\section{Acknowledgement}

Special thank goes to Tamás Budai to include the mentioned techniques in MaxWhere.

\section{References}

[1] L. Keviczky, R. Bars, J. Hetthéssy, C. Bányász, Control Engineering, Springer, Singapore, 2019.

doi:10.1007/978-981-10-8297-9_1

[2] B. Lantos, Control Systems, Theory and Design I., Academic Press, Budapest, 2009, in Hungarian.

[3] K. Ogata, Modern Control Engineering, Prentice-Hall, Upper Saddle River, New Jersey, 1997.

[4] P. Gáspár, J. Bokor, A. Soumelidis, An inverted pendulum tool for teaching linear optimal and model based control, Periodica Polytechnica Transportation Engineering 25 (1-2) (1997) pp. 9-19.

URL https://pp.bme.hu/tr/article/view/6592.

[5] V. A. Arya, E. G. Ashni, Stabilisation of cart inverted pendulum using the combination of PD and PID control, International Journal of Innovative Research in Science, Engineering and Technology 7 (4) (2018) pp. 3559-3565. 
[6] B. Messner, D. Tilbury, Control tutorials for Matlab Simulink [cited 2019-01-20]. URL http: / / ctms.engin.umich.edu/CTMS /

[7] Md. Akhtaruzzaman, A. A. Shafie, Comparative assessment and result analysis of various control methods, applied on a rotary inverted pendulum, SRV 02 series, Advances in Applied Science Research, Pelagia Research 2 (6) (2011) pp. 83-100.

[8] Scilab [cited 2019-01-20].

URL https://www.scilab.org/

[9] Mistems Ltd., MaxWhere [cited 2019-01-20].

URL https: / / www . maxwhere.com/

[10] T. Budai, M. Kuczmann, Development of a VR capable virtual laboratory framework, Pollack Periodica 13 (3) (2018) pp. 83-93.

doi:10.1556/606.2018.13.3.9.

[11] B. Lantos, M. Lőrinc, Nonlinear control of vehicles and robots, Springer-Verlag, London, 2011.

doi: $10.1007 / 978-1-84996-122-6$

[12] A. D. Drexler, Nonlinear and robust control, typescript, Budapest University of Technology and Economics, 2015, in Hungarian.

[13] W. Liuping, Model predictive control system design and implementation using Matlab, Springer-Verlag, London, 2009.

doi:10.1007/978-1-84882-331-0 